\title{
SPEECH RECOGNITION OF KV-PATTERNED INDONESIAN SYLLABLE USING MFCC, WAVELET AND HMM
}

\author{
${ }^{\mathrm{a}}$ Syahroni Hidayat, ${ }^{\mathrm{b}}$ Risanuri Hidayat, ${ }^{\mathrm{c},}$ Teguh Bharata Adji \\ a Jurusan Teknik Informatika, STMIK Bumigora Mataram \\ Jl. Ismail Marzuki, Cakranegara, Mataram, Nusa Tenggara Barat \\ ${ }^{b, c}$ Departemen Teknik Elektro dan Teknologi Informasi \\ Fakultas Teknik, Universitas Gadjah Mada \\ Jl. Grafika no.2 Yogyakarta-55281, Indonesia \\ Email : syahroni.hidayat@stmikbumigora.ac.id
}

\begin{abstract}
The Indonesian language is an agglutinative language which has complex suffixes and affixes attached on its root. For this reason there is a high possibility to recognize Indonesian speech based on its syllables. The syllable-based Indonesian speech recognition could reduce the database and recognize new Indonesian vocabularies which evolve as the result of language development. MFCC and WPT daubechies $3^{\text {rd }}(D B 3)$ and $7^{\text {th }}(D B 7)$ order methods are used in feature extraction process and HMM with Euclidean distance probability is applied for classification. The results shows that the best recognition rateis $75 \%$ and $70.8 \%$ for MFCC and WPT method respectively, which come from the testing using training data test. Meanwhile, for testing using external data test WPT method excel the MFCC method, where the best recognition rate is $53.1 \%$ for WPT and $47 \%$ for MFCC. For MFCC the accuracy increased asthe data length and the frame length increased. In WPT, the increase in accuracy is influenced by the length of data, type of the wavelet and decomposition level. It is also found that as the variation of state increased the recognition for both methods decreased.
\end{abstract}

Keywords : Indonesian Automatic Speech Recognition, Syllables, Mel Frequency Cepstral Coefficient (MFCC), Wavelet Packet Transform (WPT).. 


\section{INTRODUCTION}

Research on Automatic Speech Recognition (ASR) has achieved a very high success rate. However, this achievementhasnot cover all the languages in the world (i.e. Indonesian language). Aside from the few number of research in this area, the accuracy of the Indonesian ASR is still unsatisfactory. Research from [1]-[3] are focused on building Large Vocabulary Continuous Speech Recognition (LVCSR) of Indonesian language and still constrained by Out Of Vocabulary (OOV) problems. In [4] and [5] research are conducted on the isolated Indonesian ASR and limited to vocals and some specific vocabulary.

Indonesian language which is the native language of the Republic of Indonesia has a different pattern of language from other countries. It is not restricted by the use of gender and tonal. Thus, Indonesian language is one of agglutinative language. It means that it has complex suffixes and prefixes that attached on its root. Roots of Indonesian language have simple pattern of syllables, generally $\mathrm{V}, \mathrm{VK}$, $\mathrm{KV}$ and KVK[6], [7].

In the utterance of an Indonesian word, every syllable will be followed by a breath. This enables the pauses among syllables and produces a different representation of the sound signals of Indonesian word for each syllable. Therefore, Indonesian speech can be recognized at the level of syllables. Abriyono [7] has done an ASR research to convert the Indonesian speech into text in syllables domain, but its accuracy is very low. While Suyanto [8] proposed a novel design to develop Indonesian LVCSR system, which is based on the unification of phonemes and syllables. Recalling the fact that $92 \%$ of Indonesian vocabulariesare composed by 4 patterns of syllables as mentioned above, it is expected that a large LVCSR database can be replaced byasmaller database of syllables that would cover the whole Indonesian vocabularies

The recognition rate is influenced by the extraction and classification method used. Recently, MFCC extraction method is more widely used, as has been done by[1]-[4], [7], and [9]. The wavelet method was applied by [5], [10], and [11]. Both methods have their own advantages and disadvantages as described in [12]. The application of these two methods is also still around the phoneme, words and sentence recognition. The application of MFCC method on syllable domain has been carried out by [7] for Indonesian language. But the recognition rate is still very low. Hence, it is necessary to apply another method of extraction that may enhance the syllable recognition accuracy, namely Wavelet Packet Transform (WPT) extraction method.

As for classification, Hidden Markov Models (HMM), which works based on stochastic processes gives the best results for its ability to recognize data with small variability, as done by[1]-[3], [9]. However, the use of Gaussian Mixture Model (GMM) as the observation probability distribution model still leaves problem in computational process. Thus, Budi[9] and Buono [13] used the Euclidean distance probability as a substitute for GMM distribution probability.

This research is focused on syllable-based Indonesian speech recognition of $\mathrm{KV}$-patterned using MFCC and WPT feature extraction method. HMM by applying Euclidean distance probability method is applied for classification

\section{RESEARCH RELATED COMPONENTS}

\section{Indonesian Syllables}

Syllable is the smallest part of a word and cannot be divided. Indonesian syllables are always have a vowel as the core of a word. The core may be preceded or followed by a consonant or more. There are also words with a syllable that consists of a vowel only.

Table 1. Indonesian syllable pattern

\begin{tabular}{lll}
\hline No. & $\begin{array}{l}\text { Syllable } \\
\text { Pattern }\end{array}$ & Example \\
\hline 1. & V & E-nergi \\
2. & VK & Ar-ti \\
3. & KV & Ka-ki \\
4. & KVK & Tek-nik \\
5. & KVKK & Mo-dern \\
6. & KVKKK & Korps \\
7. & KKV & Pra-sasti \\
8. & KKVK & Trak-tor \\
9. & KKKV & Stra-tegi \\
10. & KKKVK & Struk-tur \\
11. & KKVKK & Kom-pleks \\
\hline
\end{tabular}


Some basic patterns of Indonesian syllables are shown in Table 1. Some of Indonesian syllables pattern, such as $\mathrm{V}, \mathrm{VK}, \mathrm{KV}$ and $\mathrm{KVK}$ form most of the Indonesian root vocabularies[6]-[8].

\section{Pre-emphasis}

Pre-emphasis is used to increase the amount of sound energy of the signal at its higher frequency. This process is designated to reduce the adverse effect from the transmission and background interference. In fact, for voiced signal segments such as vowels, the amount of its energy at low frequency is greater than at high frequency. A decrease in frequency energy is caused by the nature of the glottal vibration. Improving the high frequency energy will make the information from higher formants available for the acoustic models and increase the accuracy of voice detection[14]. Pre emphasis is a first order high-pass filter which is expressed by the Equation (1) [15].

$$
y[n]=x[n]-\alpha x[n-1]
$$

Where $y[n]$ and $x[n]$ are espectively the output and input signals at discrete time step $\mathrm{n}$, and $a$ is a constant with value $0.9 \leq a \leq 1$.

\section{Normalization}

Recording speech signal repeatedly will make the energy level obtained be stratified, this is due to the change in the microphone distance and signal source. Normalization of amplitude is applied to overcome the energy levels which is not consistent between signals. Thus, the quality of energy-related characteristics can be improved and all of the data that will be fed to a voice activity detection process will have the same measurement standards[5]. Normalization is expressed by the Equation (2).

$$
S_{n o}(n)=\frac{S(n)}{\max (|S(n)|)}
$$

Where, $S_{n o}(n)$ is normalized signal and $S(n)$ is input signal at discrete time step $n$

\section{Voice Activity Detection (VAD)}

Before performing the feature extraction process, it is essential to eliminate the noise that accompanies the voice signal. One way to do it is by detecting voice activity by calculating the energy of the voice signals to distinguish between noise signal and voiced signal. The signal energy is calculated by the following the Equation (3) [7].

$$
E=\frac{1}{N} \sum_{n-1}^{N}|x(n)|
$$

Where $E$ is short time signal energy, $N$ is number of signal sample, and $x$ is value of signal input at discrete time step $n$.

The VAD algorithm are describe as follows:

1. Speech signal framed with frame length (lfr) $4 \mathrm{~ms}$ and shift frame (sfr) $2 \mathrm{~ms}$.

2. Multiply each frame with rectangular window function $w_{\text {rec }}$.

3. Calculate the energy of each frame $(E F)$.

4. Determine threshold value, $T H$, by multiplying maximum value of $E F$ with specific weight value. The weight value is based on the maximum value of $E F$. If $E F_{\max }>1$ then weight $=10^{-2} / 4$, else if $E F_{\max }<1$ then weight $=10^{-1} / 4$. The weighted threshold value makes it dynamic. Therefore, the start and end point determination will be more exact.

5. Specify each frame that contains speech signal by comparing between the value of $E F$ and $T H$. If $E F>T H$ then $E F=1$, else if $E F<T H$ then $E F=0$.

6. Determine number of syllable $(S K)$, start index $\left(I_{s}\right)$ and end index $\left(I_{e}\right)$ as follow:

a. Determine $S K=0$.

b. Start from frame-2 until end, find the value of frame that fulfilled $E F(i-1)=1$ and $E F(i)=0$.

c. If fulfilled, then $S K=S K+1$ and $I_{e}=i$.

d. For star index $\left(I_{s}\right)$, determine $S K=0$.

e. Start from frame- 2 until the end, find the value of frame that fulfilled $E F(i-1)$ $=0$ and $E F(i)=1$.

f. If satisfied, then $S K=S K+1$ and $I_{e}=$ $i$. To avoid the data losses because of the indexing problem we applied this 


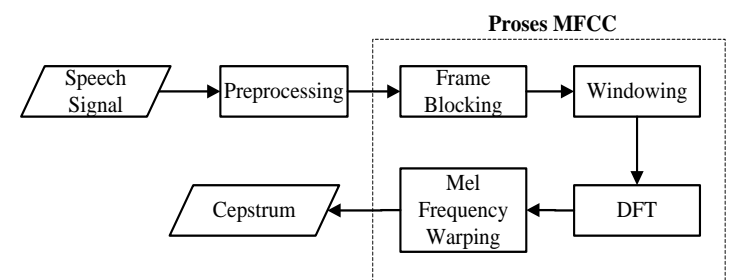

Figure 1. MFCC process

condition $I_{e}=$ number of max frame and $I_{s}=1$.

7. Determine new syllable signal $(S K B)$ as follow:

a. Determine the number of $S K$.

b. If $S K=1$, then $S K B=I_{s}{ }^{*} s f r: I_{e} *(s f r-1)$.

c. If $S K>1$, then $S K B=I_{s}(i) * s f r: I_{a}(i) *(s f r-$ 1).

\section{Mel Frequency Cepstral Coefficient (MFCC).}

MFCC feature extraction process is a process of creating vector characteristic based on the discrete Fourier transform. Its stages briefly shown in Figure 1.

Recorded speech signal enters the preprocessing stage and separated within its syllable. The separated signal is then converted into cepstrum by using MFCC process. The MFCC process consists of frame blocking, windowing, Discrete Fourier Transform and Mel frequency warping. The detail of the process in each block is described below.

\section{Frame Blocking and Windowing}

Speech signal is a non-stationary signal. It means that its statistic properties always change over time. Thus, it is not possible to extract the spectral characteristics of speech signal at once. Therefore, the spectral characteristic of speech signal extracted through a window represents parts of certain speech. As a result, it can be assumed that the speech signal is stationary[14].

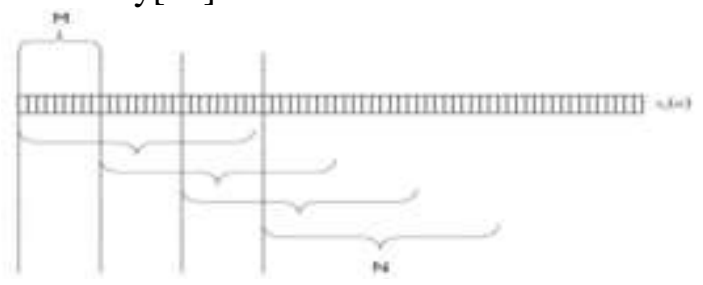

Figure 2. Process of frame blocking
The process of windowing is characterized by three parameters, namely the width of the window, the offset between the window and form of window. Windowing result is referred to the frame, with the frame size and frame shift specified in milliseconds. The range of the shifted frames is between 1/3 - 1/2 times of its frame length. Figure 2 shows the process of framing the speech signal.

Window function used is worth a maximum of 1 in the areas within the window and zero for the other areas. The window moves along the speech signal and extracting the shape of the signal inside. In this study, Hamming window function is used, it is defined by the Equation (4):

$w[n]=\left\{\begin{array}{cc}\alpha-\beta \cos \left(\frac{2 \pi n}{L}\right), & 0 \leq n \leq L-1 \\ 0 & \text { others }\end{array}\right.$

and the process of multiplying the value of signal at frames $N$ at time $n, s[n]$, with the value of the window function at the time $n$, $w[n]$ :

$$
y[n]=w[n] s[n]
$$

\section{Discrete Fourier Transform}

Discrete Fourier Transform (DFT) is used to extract spectral information from the window signal to determine the energy contained in different frequency bands[9]. DFT is expressed in the Equation (6).

$$
X[k]=\sum_{n=0}^{N-1} x[n] e^{-j 2 \pi n k / N}
$$

Where $X$ is the DFT process output, $N$ is number of signal sample, $x$ is input signal at discrete time step $n$ and $k$ is $1,2,3 \ldots, N-1$. Since the result of Fourier transform is symmetric, it is only half of it that will be used for further process.

\section{Mel Frequency}

Mel Frequency Warping is the process of wrapping signal spectrum using triangular filter bank. Since the speech signal is different with the human auditory perception, where speech signal does not have a linear frequency scale, 
an adjustment with the human auditory perception which is linear is needed in the feature of extraction process in order to improve recognition performance. As a reference, the frequency scale in $\mathrm{Hz}$ and $\mathrm{Mel}$ scale is linear at frequencies below $1000 \mathrm{~Hz}$ and logarithmic in frequency above it. To change the frequency of the speech into frequency Mel following equation is used[15]:

$$
\operatorname{mel}(f)=1125 \ln \left(1+\frac{f_{H z}}{700}\right)
$$

while the inverse is:

$$
f_{H z}(m e l)=700\left(\exp \left(\frac{m e l}{1125}\right)-1\right)
$$

After the midpoint of frequency is obtained, the process continued with creating $M$ triangular filters by using Equation (9):

$$
H_{m}[k]=\left\{\begin{array}{cc}
0 & k<f[m-1] \\
\frac{(k-f[m-1])}{(f[m]-f[m-1])} & f[m-1] \leq k \leq f[m] \\
\frac{(f[m+1]-k)}{(f[m+1]-f[m])} & f[m] \leq k \leq f[m+1] \\
0 & k>f[m+1]
\end{array}\right.
$$

The shape of triangular filter bank, based on equation (9) above, is shown in Figure 3.

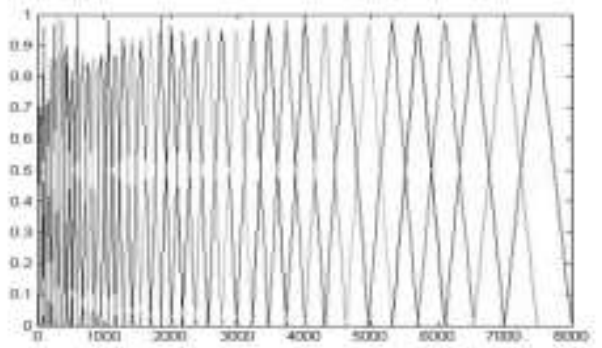

Figure 3. Triangular filter bank

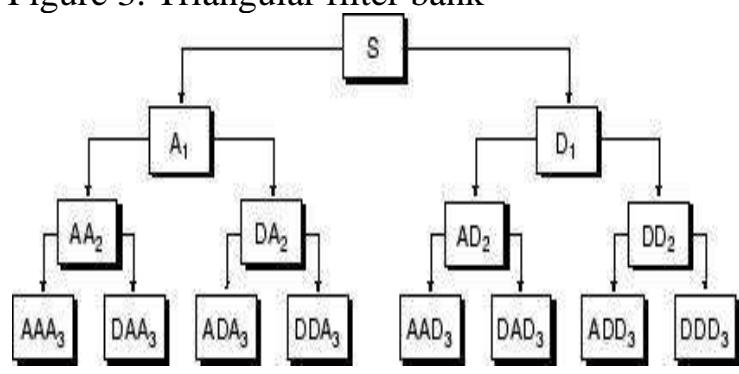

Figure 4. Decomposition process of WPT[16]

\section{Discrete Cosine Transform}

At MFCC stage, cepstrum process is the process of transforming the Mel frequency back into time domain. Output of this process is the Mel Cepstrum Coefficient (MFCC). Since Mel spectrum coefficient is a real number, it can be inversed back into time domain by using Discrete Cosine Transform (DCT) as seen in Equation (10) [15]:

$$
c[n]=\sum_{m=0}^{M-1} S[m] \cos \left(\frac{\pi n\left(m+\frac{1}{2}\right)}{M}\right) \quad 0 \leq n<M
$$

Where $S[m]$ is the output of multiplying the spectrum by its conjugate.

\section{Wavelet Packet Transform (WPT)}

Wavelet is a short wave with limited duration which has an average value of zero. This wavelet concentrates the energy in space and time that is suitable for analyzing a temporary signal. There are two types of wavelet namely continuous wavelet transform and discrete wavelet transform[5]. WPT is one of the development of the discrete wavelet transform which decomposes both sides of approximation and detail. WPT decomposition process is shown in Figure 4, and the decomposition equation is shown in (11) and (12):

$$
\begin{aligned}
& y_{h}[k]=\sum_{n} x[n] h[2 k-n] \\
& y_{l}[k]=\sum_{n} x[n] \operatorname{g}[2 k-n]
\end{aligned}
$$

Where $y_{h}[k]$ is the detail of signal information, $D$, and $y_{l}[k]$ is rough approximation of scaling function, $A . x[n]$ is an original signal with $h[n]$ an $g[n]$ are respectively HPF coefficient and LPF coefficient.

WPT decomposition process will produce sub-band as much as $2 \mathrm{j}$ where $\mathrm{j}$ is the level of decomposition. To create a feature vector, the energy level of each sub-band is calculated by using the Equation (13):

$$
E_{i}=\sqrt{\sum_{k=1}^{N}\left|X_{i}(k)\right|^{2}}
$$


Energy normalization is necessary so that the value of feature vector obtained has the same standards. Energy normalization is done by dividing the total energy of each sub-band to the total energy sub-bands used. It is formulated by the Equation (14) and Equation (15).

$$
\begin{gathered}
E_{\text {tot }}=\sqrt{\sum_{i=1}^{I} E_{i}^{2}} \\
V_{\text {energi }}=\frac{E_{i}}{E_{\text {tot }}}
\end{gathered}
$$

\section{Hidden Markov Models (HMM)}

Hidden Markov Models is based on a double stochastic process, the first is a stochastic process that produces a state that cannot be observed, and the second is the process that produces cascading observation that can be observed[17]. There are two types of HMM, Ergodic and left-right. HMM leftright is used to model the speech signal because the speech cannot be repeated to the previous state.

An HMM is modeled by the following elements:

$$
\lambda=(A, B, \pi)
$$

And the left-right HMM type model is described as follows:

$A=\left\{a_{i j}\right\}$, state transition probability:

$$
\begin{aligned}
& a_{i j}=0 \text { for } j<i \text { and } j>i+1, a_{N N}=1, \\
& a_{N j}=0, j<N .
\end{aligned}
$$

$\pi=\left\{\pi_{i}\right\}$, prior probability state distribution:

$$
\pi_{i}=1 \text { for } i=1, \text { dan } \pi_{i}=0 \text { for } i \neq 0
$$

$B=b_{j}(k), \quad$ observation probability distribution

Generally, the observation probability distribution of HMM is modeled by Gaussian Mixture Model (GMM). However, this modeling brings a computational problem, therefore, Euclidean distance probability can be used as an alternative which its equation is expressed by:

$$
\begin{gathered}
b_{j}\left(o_{t}\right)=\frac{1}{1+d_{j}\left(o_{t}\right)} \\
d_{j}\left(o_{t}\right)=\sqrt{\sum_{k=1}^{M}\left(o_{t k}-\mu_{j k}\right)^{2}}
\end{gathered}
$$

\section{METHOD}

The data used in this research is recording file of Indonesian syllable voices which was taken from 8 male speakers. Speech data which recorded from 6 speakers is used as training data set. The testing data set is taken from the two remaining speakers plus one whom voice was included in training data set. Recording process is done byusing Matlab R2009a in open area. The duration of recording was 1 second with 5 times repetitions for syllable utterance and 5 seconds with 1 time repetition for word utterance. The frequency sampling used in recording is $16000 \mathrm{~Hz}, \mathrm{PCM}-16$ bit, mono and stored in *.wav format. The syllables and word used in this research respectively have $\mathrm{KV}$ and $\mathrm{KV}-\mathrm{KV}$ pattern as shown in

Table 2 and Table 3 . There are 24 syllables recorded in total with 5 times repetitions. It means that 720 syllable data in accumulate used as training data, each syllable is represented by 30 data records. As for testing, there are 72 of syllable data records in total. Meanwhile, for word data records are 48 in total. If word data separated by its syllables, it means that there are 96 of syllable data records.

Table 2. Syllable for training and testing data

\begin{tabular}{ccccccc}
\hline & \multicolumn{6}{c}{ Training Data } \\
\cline { 2 - 7 } & a & i & u & e & e2 & o \\
\hline g & ga & gi & gu & ge & ge2 & go \\
k & ka & ki & ku & ke & ke2 & ko \\
l & la & li & lu & le & le2 & lo \\
r & ra & ri & ru & re & re2 & ro \\
\hline
\end{tabular}

Table 3. Word testing data set

\begin{tabular}{clll}
\hline \multicolumn{4}{c}{ Testing Data } \\
\hline guuruu & guuru & guruu & guru \\
kaakii & kaaki & kakii & kaki \\
laaguu & laagu & laguu & lagu \\
raagaa & raaga & ragaa & raga \\
\hline
\end{tabular}




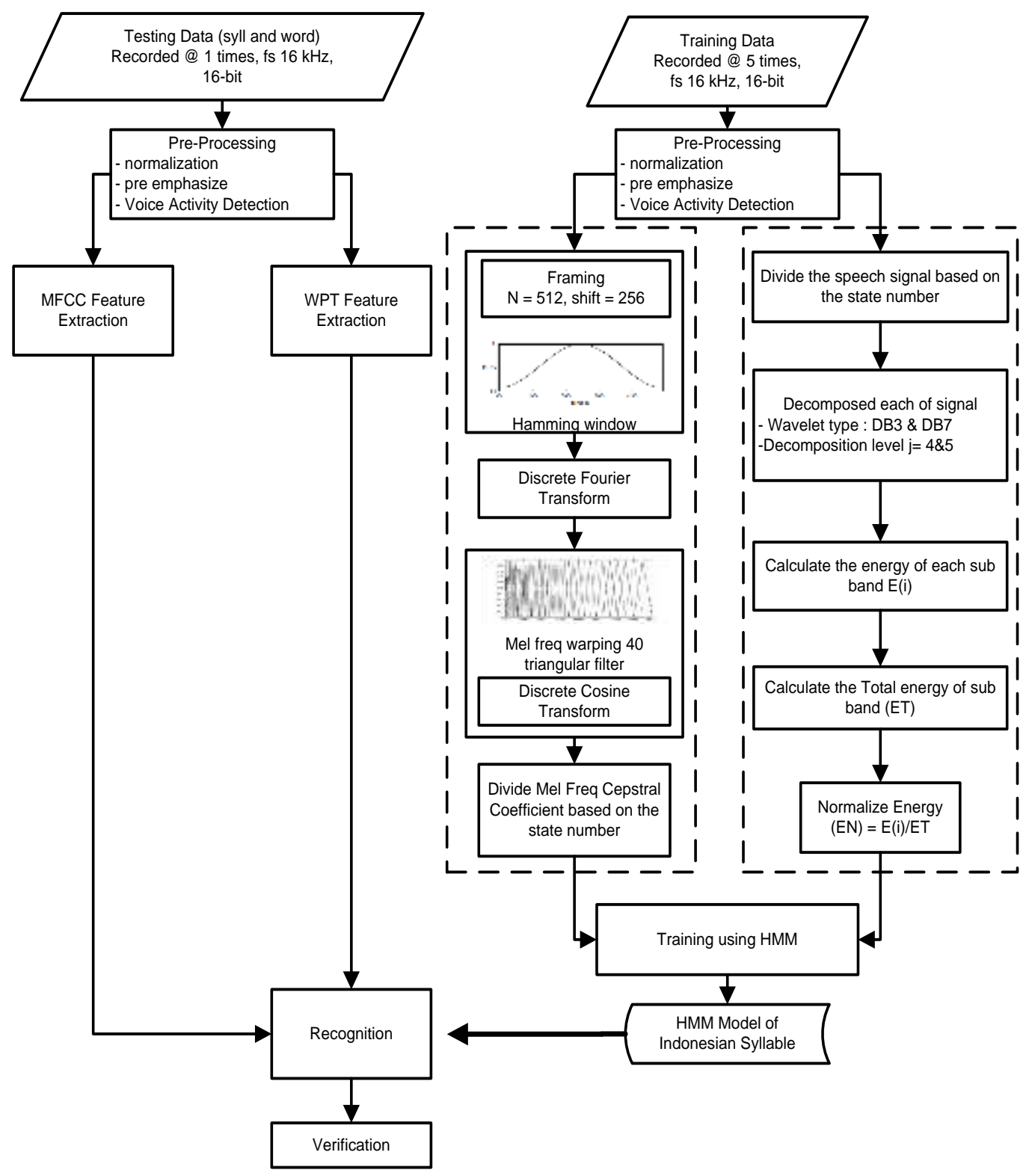

Figure 6. Training and testing process

VAD algorithm is used to separate the speech signal from its noise. It is integrated with preemphasis and normalization process. The output of this process is emphasized on speech signal with different length. Therefore, the length of output signal will be equalized explicitly to 1024 and 2048 samples. It is based on research conducted by Farooq [10] which said that length of English syllable is $32 \mathrm{~ms}$ in $16000 \mathrm{~Hz}$ of frequency sampling.

Thus, the selection of that length is long enough to represent the whole Indonesian syllable signals. In adition, the computational process would be more efficient by using this process. Next step is extraction of speech sample by using MFCC and WPT feature extraction methods. In MFCC feature extraction process, these variables are applied. The length of frame is for about 512 samples with $50 \%$ overlap among its frames. To get the cepstrum of 40 triangular, filter bank is used in Mel warping process. The cepstrum is then inversed by using DCT to obtain Mel cepstrum coefficient or MFCC. The Mel coefficient used 
in next stages is respectively taken from 3rd coefficient to 14 th coefficient. Therefore, there are 12 numbers of coefficients in total.

As for WPT feature extraction, Daubechies 3rd (DB3) and 7th (DB7) order are applied by using decomposition level $\mathrm{j}=4$ and $\mathrm{j}=5$. The feature vector then is created by calculating the normalized energy of each sub-band decomposition result. The number of sub-band created in every level of decomposition is the same as $2 \mathrm{j}$ coefficient of feature vector. The feature vector of each method is then classified by using HMM with Euclidean distance probability as its distribution probability model. Numbers of state used are 2 and 3 states. Number of state of 2 is suitable with number of consonant and vowel of syllable pattern, while number of state of 3 represents the consonant and vowel and the transition of them.

The testing data set used for evaluation process is arranged in training data set of syllable (DLSK), testing data set of syllable (DUSK) and testing data set of word (DUK). The classification result will be labeled as 1 if true and labeled as 0 if it is not suitable with the reference. The accuracy is calculated by dividing the number of true classification with the total number of syllable reference. In the end, the MFCC and WPT recognition rate will be compared. As for variable of data length, frame length, number of state, level of decomposition and the type of wavelet are analyzed to know which variable influencing the recognition rate.

\section{RESULT AND DISCUSSION}

\section{Recognition Rate using MFCC and WPT Method}

The recognition rate of Indonesian language speech recognition based on syllable recognition rate result by using MFCC feature vector is shown at Figure 7 and Figure 8. The graph represents the testing result using training data set of syllable (DLSK), testing data using testing data set of syllable (DUSK) and testing data set of word (DUK). In particular for the recognition result of the test using testing data set is represented by each speaker. Where P1, P2 and P3 respectively are representation of first, second and third speaker. As for P3 is speaker whom his speech data records included in training data set feature, length of 1024 samples.

The recognition rate of MFCC feature for 1024 samples of data length is shown in Figure 7. DLSK best recognition result is $71 \%$, however it is not followed by DUSK and DUK recognition result. The best accuracy for DUSK and DUK are respectively $25 \%$ and $22 \%$.

Figure 8 shows the recognition rate of MFCC using 2048 samples of data length. For this case the best recognition rate for DLSK has increased to $75 \%$. For DUSK and DUK, their best recognition rate has increased as well around $38 \%$ and $47 \%$ respectively.

Figure 9 and figure 10 shows the recognition result of syllable using WPT DB3 feature vector. The recognition rate of 1024 samples of data length (Figure 9), the best recognition rate for DLSK is $54.2 \%$. As for both DUSK and DUK, their best recognition rates are respectively $37.5 \%$ and $46.9 \%$. In addition, the best recognition rates with 2048 samples of data length (Figure 10) for DLSK, DUSK and DUK are $62.5 \%, 33.3 \%$ and $50 \%$ respectively.

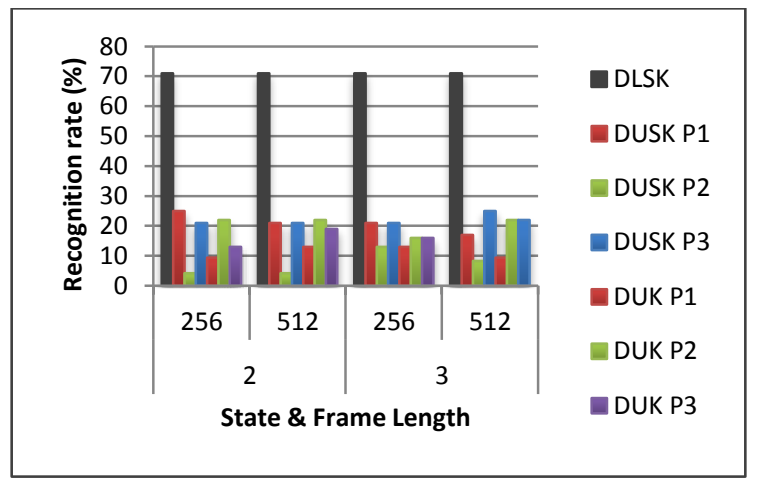

Figure 7. The recognition rate of MFCC feature, length of 1024 samples

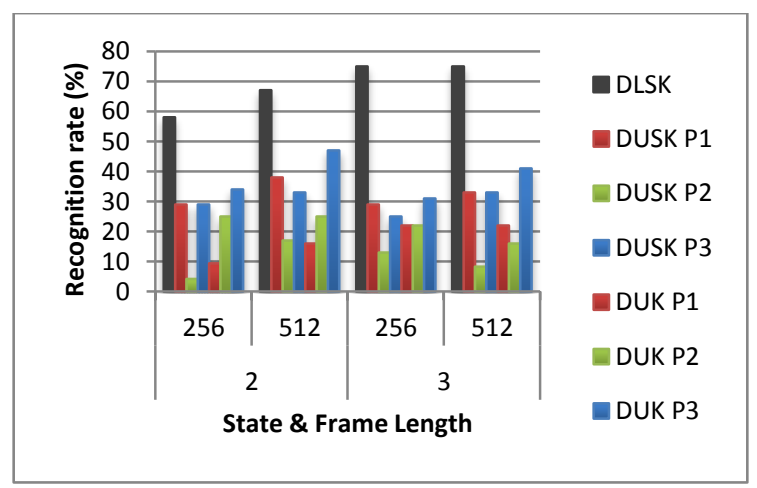

Figure 8. The recognition rate of MFCC feature, length of 2048 samples 


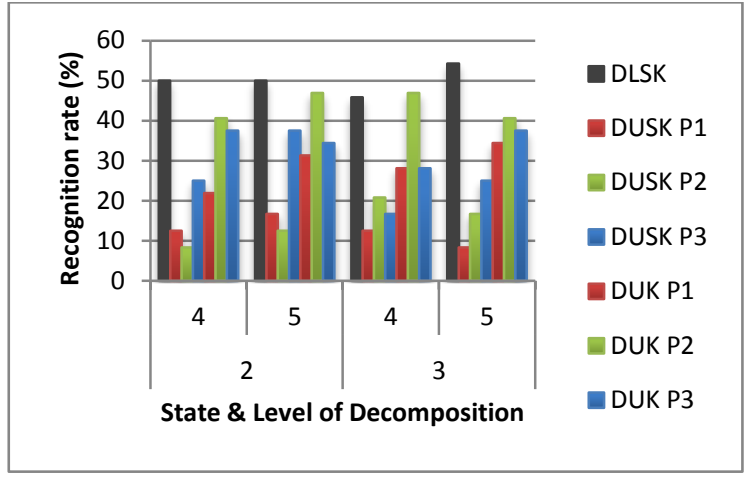

Figure 9. The recognition rate of WPT DB3 feature, length of 1024 samples

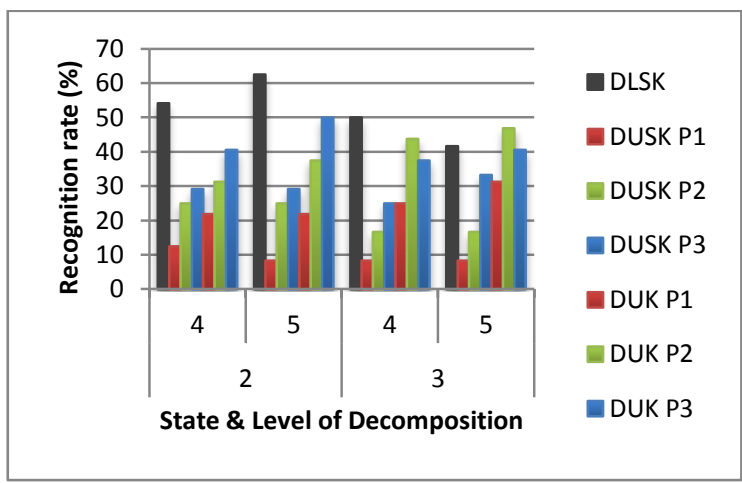

Figure 10. The recognition rate of WPT DB3 feature, length of 2048 samples

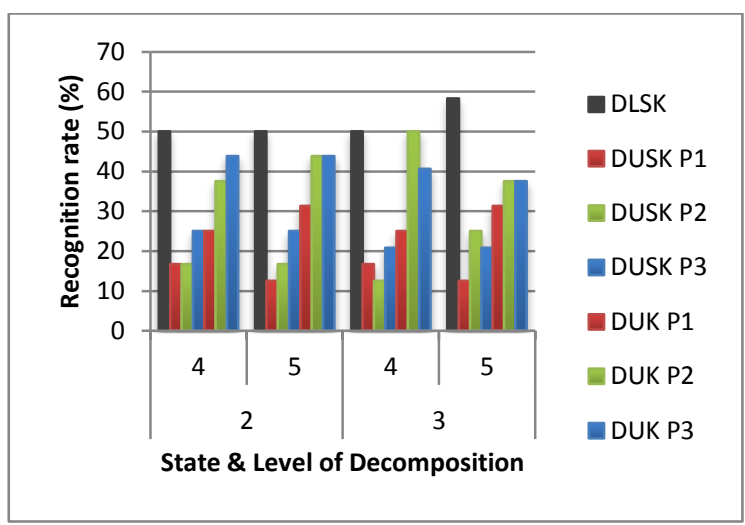

Figure 11. The recognition rate of WPT DB7 feature, length of 1024 samples

Furthermore, the recognition rate for WPT DB7 by using 1024 samples of data length is shown in Figure 11. The best recognition result for DLSK is $58.3 \%$, for DUSK is $25 \%$ and for DUK is $50 \%$. As the recognition rate for WPT DB7, Figure 12, with 2048 samples of data length is $70.8 \%$ for DLSK, $29.2 \%$ for DUSK and $53.1 \%$ for DUK.

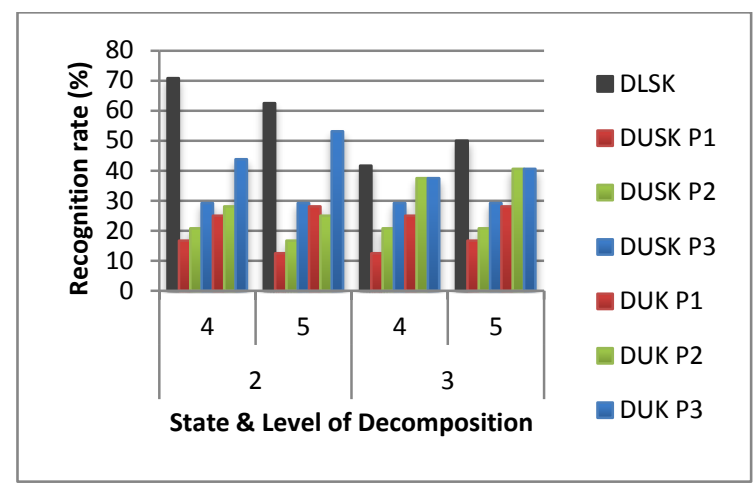

Figure 12. The recognition rate of WPT DB7 feature, length of 2048 samples

From discussion above, it can be seen that the usage of each WPT DB7 and WPT DB3 features has some advantages in recognition of syllable. For testing using DLSK and DUK, the WPT DB7 feature usage is better than WPT DB3 feature. However, at testing using DUSK, WPT DB3 feature usage overcomes the WPT DB7 feature.

Focusing on speaker effect, the usage of WPT feature vector, P3 has always had a higher recognition rate than $\mathrm{P} 1$ and $\mathrm{P} 2$. However in special case, P1 and P2 could equalize even surpass the recognition rate of P3. It explains that the usage of WPT feature is better than MFCC feature, primarily for testing by using speech data set without prior training.

The Influence of Data Length, Frame Length, Number of State, Level of Decomposition and Wavelet Type

In this part, the discussion will be on the influence of data length, state, frame length, level of decomposition and the type of wavelet used in recognition rate. The analysis focused on the best recognition result of each testing data set.

The influence of data length to the recognition rate is shown in Figure 13. It can be seen form the figure that the recognition rate is increased when the length of data is added, especially when it is applied at MFCC feature vector. The feature extraction of MFCC which is based on DFT process has weakness in mapping the signal into time-frequency domain. It is influenced by the framing and windowing algorithm used in Fourier transform process. Both algorithm makes time sampling process of DFT more rigid and has high possibility in losing the information. 


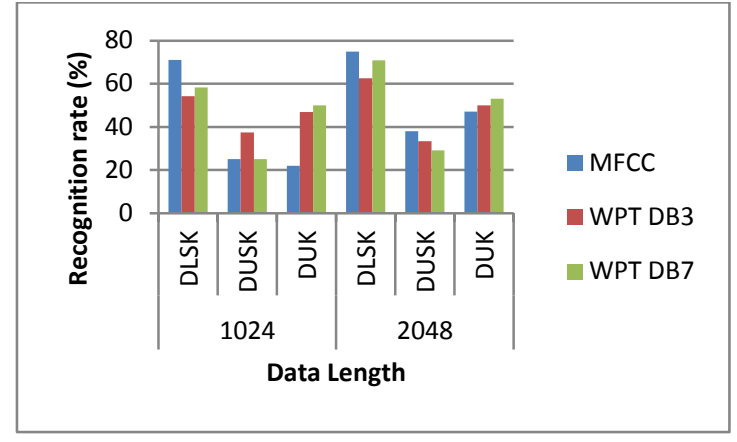

Figure 13. Influence of data length on accuracy

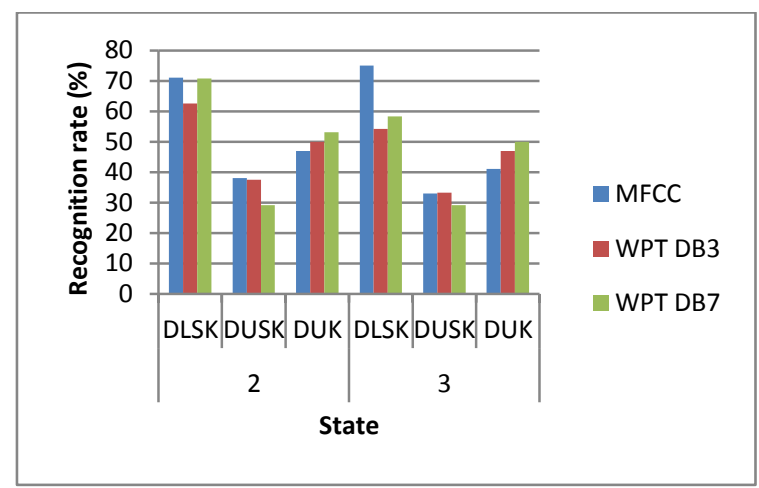

Figure 14. Influence of number of state on accuracy

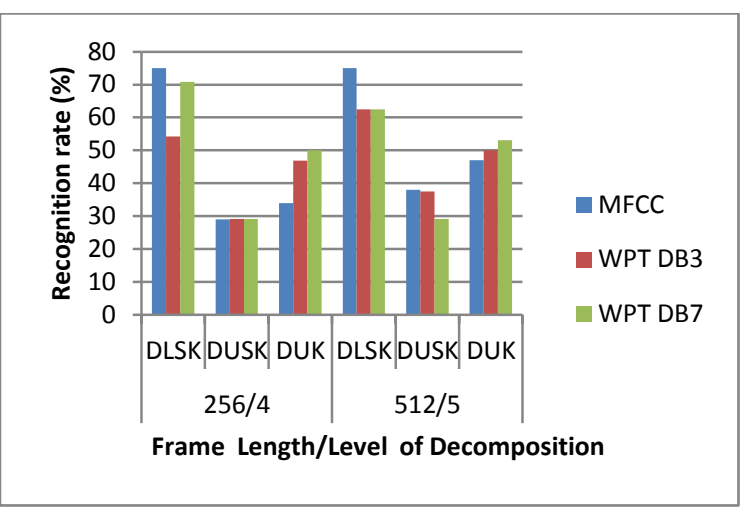

Figure 15. Influence of frame length/ level of decomposition on accuracy

The effect of number of state to recognition rate can be seen from Figure 14, where the addition of number of state decreases the recognition accuracy. For a KV pattern of syllable, it is adequate to use 2 states in classification process of HMM because each state already represents the characteristic of consonant and vowel respectively. However, the usage of larger number of state is necessary when processing the syllable with higher pattern. In addition, it can be applied to analyze the transition of phoneme consonant to phoneme vowel. In this case, the usage of 2 states gives a better recognition result. For the influence of frame length and level of decomposition to recognition rate, it can be seen in Figure 14, both frame length in MFCC feature extraction process and level of decomposition in WPT feature extraction process give positive effect, where the recognition rate is increased as both variables increased. As it was known before, frame length and level of decomposition influence the signal mapping into frequency and time domain.

In MFCC process, the usage of frame length with 512 samples is better than 256 samples. The bigger the frame length the better the energy resolution of speech signal in frequency domain. However, the frame length with 512 samples length cannot be applied to short time speech signal like consonant.

As for the WPT process, a greater level of decomposition will influence the energy level and the number of feature vector of speech signal. The higher the level of decomposition used, the more various the characteristic of speech feature vector will be and the recognition rate will increase. However, it is not recommended to use too high level of decomposition because the down sample process can omit important information of the speech signal. Thus, based on this study, the WPT with 5th level of decomposition would be better if it is applied in Indonesian syllable recognition.

The level of decomposition influence cannot be separated from the type of waveled used. It is shown from Figure 12 to Figure 15, the best recognition rate are mostly obtained from applying wavelet DB7 rather than wavelet DB3, especially in recognition by using training data set of syllable (DLSK) and testing data set of word (DUK). However, in tests using testing data set of syllables (DUSK), wavelet DB3 has always bring better results. It is because the base function of wavelet DB3 is more similar to the syllable signal which recorded directly. Meanwhile, the base function of wavelet DB7 is more similar to the syllables signal which extract from the word-recorded signal.

As a result, it can be generally concluded that in syllable-based Indonesian speech recognition, for the testing using training data set, MFCC method is better than WPT method. 
However, for testing using testing data set, the recognition rate of WPT method overcomes the MFCC method. Thus, applying WPT method in recognition of Indonesian syllable is better than MFCC. As for type of wavelet used, wavelet Daubechies DB7 gives more better result than wavelet DB3, but it does not applicable to all circumtances.

\section{CONCLUSION}

From this research, it can be concluded that:

1. The syllable-based Indonesian speech recognition of KV-patterned using MFCC and WPT as feature extraction method and HMM with Euclidean distance probability as classifier have been studied.

2. The best recognition rate for MFCC method is $75 \%$ for testing using syllables training data set (DLSK), 38\% for testing using syllables testing data set (DUSK) and 47\% for testing using word testing data set (DUK). As for WPT method, the recognition rate is $70.8 \%$ for testing using syllables training data set (DLSK), 37.5\% for testing using syllables testing data set (DUSK) and $53.1 \%$ for testing using word testing data set (DUK). WPT method is better than MFCC method when it is applied in syllable-based Indonesian speech recognition.

3. Using signal with 2048 samples length gives a better result than signal with 1024 samples length. It is because in MFCC process, the recognition rate is limited by the use of frame blocking process. As for WPT, this variable does not give an effect, because WPT simultaneously mapped the signal into time-frequency domain.

4. The usage of 5thlevel of decomposition feature vector gives a higher recognition rate than 4thlevel of decomposition feature vector. It is because the additional level of decomposition enriches the variation of syllable feature vector. For the type of wavelet Daubechies, the usage of WPT DB7 feature vector gives a better recognition than WP DB3.

5. In MFCC method, the recognition rate will increase as the length of sample and the length of frame increased. As for WPT, the recognition rate improvement is influenced by the length of sample, level of decomposition and type of wavelet used.

\section{REFERENCES}

[1] S. Sakti, E. Kelana, H. Riza, and S. Sakai, "Development of Indonesian Large Vocabulary Continuous Speech Recognition System within A-STAR Project," in TCAST, 2008, pp. 19-24.

[2] D. P. Lestari, K. Iwano, and S. Furui, "A Large Vocabulary Continuous Speech Recognition System for Indonesian Language," in 15th Indonesian Scientific Conference in Japan Proceedings, 2006, pp. 17-22.

[3] V. Ferdiansyah and A. Purwarianti, "Indonesian Automatic Speech Recognition System Using English-Based Acoustic Model," Am. J. Signal Process., vol. 2, no. 4, pp. 60-63, Aug. 2012.

[4] U. Sutisna, "Pengenalan Tutur Kata Terisolasi Menggunakan MFCC dan ANFIS," Universitas Gadjah Mada, 2013.

[5] A. Asni B, "Ekstraksi Ciri Dan Pengenalan Tutur Vokal Bahasa Indonesia

Menggunakan Metode Discrete Wavelet Transform (DWT) dan Dynamic Time Warping (DTW)," Universitas Gadjah Mada, 2014

[6] H. Alwi, S. Dardjowidjojo, H. Lapoliwa, and A. M. Moeliono, Tata Bahasa Baku Bahasa Indonesia : Edisi Ketiga. Jakarta: Balai Pustaka, 2014.

[7] Abriyono and A. Harjoko, "Pengenalan Ucapan Suku Kata Bahasa Lisan Menggunakan Ciri LPC, MFCC, dan JST," IJCCS, vol. 6, no. 2, pp. 23-34, 2012.

[8] Suyanto and S. Hartati, "Design of Indonesian LVCSR Using Combined Phoneme and Syllable Models," in The 7th International Conference on Information \& Communication Technology and Systems (ICTS), 2013, pp. 191-196.

[9] B. Darmawan, "Ekstraksi CIri Suara Untuk Pengenalan Pembicara 
Menggunakan MFCC dan Hidden Markov Models," Universitas Gadjah Mada, 2011.

[10] O. Farooq and S. Datta, "Phoneme recognition using wavelet based features," in Information Sciences, 2003, vol. 150, no. 1-2, pp. 5-15.

[11] B. T. Tan, M. F. M. Fu, A. Spray, and P. Dermody, "The Use of Wavelet Transforms in Phoneme Recognition," Proceeding Fourth Int. Conf. Spok. Lang. Process. ICSLP '96, vol. 4, 1996.

[12] M. A. Anusuya and S. K. Katti, "Front end analysis of speech recognition: a review," Int. J. Speech Technol., vol. 14, no. 2, pp. 99-145, Jan. 2011.

[13] A. Buono and B. Kusumoputro, "Pengembangan Model HMM Berbasis Maksimum Lokal Menggunakan Jarak Euclid Untuk Sistem Identifikasi
Pembicara.pdf," in National Conference on Computer Science \& Information Technology, 2007, pp. 49-54.

[14] D. Jurafsky and J. H. Martin, Speech and Language Processing: An Introduction to Natural Language Processing, Computational Linguistics, and Speech Recognition. Prentice Hall, 2008.

[15] X. Huang, A. Acero, and H.-W. Hon, Spoken Language Processing: A Guide to Theory, Algorithm and System Development. Prentice Hall, 2001.

[16] M. Misiti, Y. Misiti, G. Oppenheim, and J. Poggi, Wavelet Toolbox TM 4 User' $s$ Guide. 2009.

[17] L. R. Rabiner, "A tutorial on hidden Markov models and selected applications in speech recognition," Proceedings of the IEEE, vol. 77, no. 2. pp. 257-286, 1989. 